\title{
An ADS-B Trajectory Segmentation Correction Algorithm Based on Improved Kalman Filtering
}

\author{
Boyuan Gong, Wenbo Li and Zhiyuan Shen \\ College of Civil Aviation, Nanjing University of Aeronautics and Astronautics, Nanjing, China. 210016
}

\begin{abstract}
The Automatic Dependent Surveillance-Broadcast (ADS-B) with the advantage of faster update speed of the data, wider range of detection and sharing of traffic information. It has been regarded as one of the future mainstream surveillance systems by the International Civil Aviation Organization. This paper presents the conception, development and application of ADS-B. It also analyses the feature of the radar trajectory and ADS-B trajectory. Using CA/CV model, a new method based on improved Kalman filter to correct ADS-B trajectory is proposed to evaluate the surveillance performance based on trajectory segmentation, which is able to assess the integrity and accuracy of ADS-B data. The simulation results demonstrate the effectiveness of the proposed method.
\end{abstract}

Keywords-component; ADS-B; surveillance systems; trajectory correction; segmentation; $C A / C V$ model

\section{INTRODUCTION}

With the development of the aviation industry, air traffic flow is in a rapid growth. International Civil Aviation Organization(ICAO) has put forward a new concept of "free flight", at the request of this new concept, it becomes increasingly important to improve the flight surveillance accuracy. Automatic Dependent Surveillance - Broadcast(ADSB) provides an efficient solution to this problem, however, there's several issues during ADS-B data transmission progress, such as data loss, interference signal, and other issues. Therefore, assessing the surveillance quality of this new technology has become an important research topic. Based on previous research, this paper put forwards the research on the reliability, accuracy and integrity of ADS-B information to reduce the data error by dividing the whole path into several parts.

Automatic dependent surveillance - broadcast (ADS-B) is a surveillance technology in which an aircraft determines its position via satellite navigation and periodically broadcasts it, enabling it to be tracked. The information can be received by air traffic control ground stations as a replacement for secondary radar as no interrogation signal is needed from the ground. It can also be received by other aircraft to provide situational awareness and allow self separation.

\section{DEVELOPMENT STATUS}

ICAO's $11^{\text {th }}$ Air Navigation Conference identified ADS-B as the main direction for future surveillance technology development, and the international aviation community is actively promoting the use of the technology, some countries have put it into use.

Since 1992, USA has carried out ADS-B early application research, continue to promote the application and development of ADS-B, and widely promote the surveillance system based on ADS-B technology in Alaska, Mexico Gulf and Hawaii. The first use of ADS-B is American General Aviation. The United States made ADS-B available in 2010, and covered all America in 2013. Europe is the origin of ADS-B, it participated in ADS$\mathrm{B}$ data link assessing program at the beginning of $21^{\text {th }}$ century. In 2004, EUROCONTROL has issued a European policy on the implementation of new navigation technology and has developed the "European Civil Aviation Commission to survive the new communication and surveillance technology to promote the integration of air traffic control" implementation project, and ADS-B is the core of the project. Chinese Civil Aviation began researching ADS-B system in 1998, a new system flight path(L888), which based on ADS-B technology put into use in 2000 after systematic assessment and experiment. In 2011, they made experimental flight in flight path L642 and M771.

\section{The Evaluation Method of ADS-B Reliability}

Nowadays, Radar system is still the main tool of Air Traffic Control system. After several decades of development, the sensor has been greatly improved, but because of some shortcomings of its own, it cannot adapt to the rapid growth of traffic flow in the future. Radar system is divided into Primary Surveillance Radar(PSR) and Secondary Surveillance Radar(SSR), PSR uses echolocation method, and SSR uses the way of inquiry and response, which is made of ground interrogator and airborne responder, SSR's data sampling cycle is 4-12 seconds and is fixed, however, ADS-B receives the information data in the form of passive reception, and the navigation information obtained by the airborne equipment on the aircraft is automatically sent to other surrounding aircraft and ground receiving and processing systems via the data link. Its sampling cycle is generally 1 second, and can be artificially set according to air traffic flow. ADS-B data transmission channel for the satellite data link VHF data link, to provide more accurate than the radar data positioning information. As a result, ADS-B will become a trend for future surveillance systems.

Currently, ADS-B data contains not only aircraft identification and current four-dimensional position information, but also contains speed, expected route trends and other information. ADS-B as a non-independent surveillance (related surveillance), its reliability and independence is not as good as radar surveillance. Although with the development of new navigation systems, ADS-B has been put into use, in most cases it can only be used as backup system data or as a supplement and revision of radar data. Therefore, the study of ADS-B's reliability is particularly important and critical. 
The most important thing about tracking and surveillance systems for aircraft is to solve the filtering problem. The Kalman filter algorithm is one of the best accepted target state estimation methods. The Kalman filter performs state estimation by the current one of the measured values and the predicted value of the previous cycle. The measurement results are related to the statistical characteristics of state noise and measurement noise (zero mean and covariance), state initial conditions and so on. And these quantities are unknown and need to be assumed. Once the hypothetical model is very similar to the true value, and with the increase in filter time, there will be filtering divergence phenomenon, the difference will grow infinitely with the passage of time, the filter will lose its meaning.

Kalman filter algorithm is a linear mean square error criterion under the filter. It is used to smooth in the past moments and present moments, and predict the position, speed and acceleration of the target in the future.

At time $\mathrm{k}$, the state is estimated as $\hat{X}_{k \mid k}$, The state is further predicted

$$
\hat{x}_{k+1 \mid k}=F_{k} \hat{x}_{k \mid k}+G_{k} u_{k}
$$

Covariance is further predicted

$$
v_{k+1}=z_{k+1}-\hat{z}_{k+1 \mid k}
$$

Prediction of the measurement

$$
P_{k+1 \mid k}=F_{k} \cdot P_{k \mid k} \cdot F_{k}^{T}+Q_{k}
$$

New information

$$
\hat{z}_{k+1 \mid k}=H_{k+1} \cdot \hat{x}_{k+1 \mid k}
$$

News covariance

$$
S_{k+1}=H_{k+1 \mid k} \cdot P_{k+1 \mid k} \cdot H_{k+1}^{T}+R_{k+1}
$$

The size of the covariance reflects the degree of uncertainty, the smaller the value, the more accurate the measurement

Filter gain:

$$
K_{k+1}=P_{k+1 \mid k} \cdot H_{k+1}^{T} \cdot S_{k+1}^{-1}
$$

State filter update

$$
\hat{x}_{k+1 \mid k+1}=\hat{x}_{k+1 \mid k}+K_{k+1} \cdot v_{k+1}
$$

Covariance filter update:

$$
P_{k+1 \mid k+1}=P_{k+1 \mid k}-K_{k+1} \cdot S_{k+1} \cdot K_{k+1}^{T}
$$

Civil aviation aircrafts in the actual flight do random maneuvers due to weather conditions or controller instructions, such as turning, adjusting the height and so on. Establishing a realistic target tracking model is one of the keys to improving ADS-B surveillance accuracy. This paper presents a method of assessing the reliability of the route and route segmentation, which is particularly important and critical. According to the characteristics of different segments on the route, the $\mathrm{CA} / \mathrm{CV}$ model with the most realistic situation is selected, and the reliability, integrity and accuracy of the ADS-B surveillance data on the route route are evaluated in stages. And the reliability, integrity and accuracy of each segment constituting the route are weighted to quantify the quality of the surveillance of ADS-B on the route.

\section{ADS-B TRAJECTORY CORRECTION}

Track correction is divided into three parts, model establishment, filtering processing and reliability-related indicators.

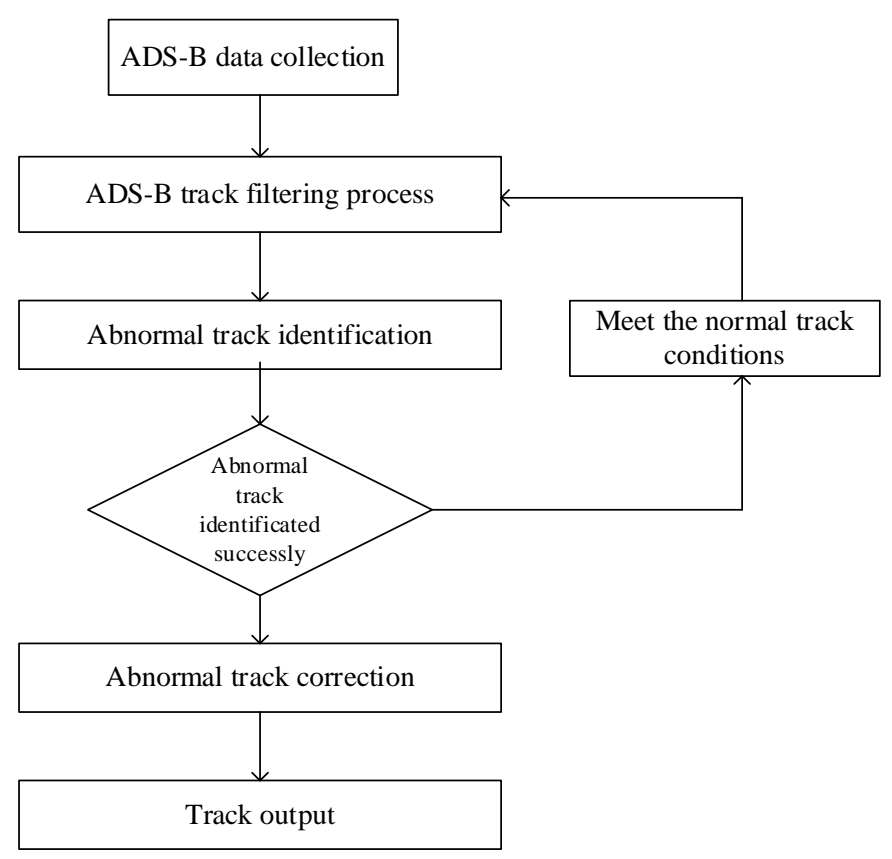

FIGURE I.FLOW DIAGRAM OF ADS-B TRAJECTORY CORRECTION

\section{A. Model Establishment}

Based on the idea of building blocks in complex adaptive system theory, the concept of basic flight model conforming to flight operation characteristics is proposed. The height and velocity profiles of the aircraft flight path are combined by a series of basic flight models (the basic flight profile of a track segment is shown in Figure. The basic flight model has the following five modes: constant velocity (CV), coordinate turn (CT), constant acceleration (CA),

$\mathrm{CV}$ : Direct flight in the main cruise segment, and constant speed climb / descent flight 
CA: the same heading and altitude in the acceleration and deceleration mode

For the above basic flight model, CV and CA is regarded as the main flight mode for short track correction. In the preparation of the program, the flight path in accordance with the flight profile characteristics and two flight patterns match.

Flight mode state equation:

Flight mode state equation is the basis of the ADS-B track correction, and different flight modes correspond to different state equations. The existing flight pattern has its corresponding state estimation equation, which can be expressed specifically as:

$$
\begin{array}{r}
x(k+1)=F_{C V} x(k)+\omega(k) \\
F_{C A}=\left[\begin{array}{ll}
1 & T \\
0 & 1
\end{array}\right], x=\left[\begin{array}{ll}
x & x
\end{array}\right]^{T}
\end{array}
$$

$x(k)$ represents the state vector of the aircraft at time $\mathrm{k} ; \mathrm{Fj}$ represents the state coefficient moment of flight mode $j, W(k)$ represents the white noise sequence of the discrete time, and $\mathrm{T}$ is the time period.

$$
\begin{gathered}
x(k+1)=F_{C A} x(k)+\omega(k) \\
F_{C A}=\left[\begin{array}{ccc}
1 & T & T^{2} / 2 \\
0 & 1 & T \\
0 & 0 & 1
\end{array}\right], x=\left[\begin{array}{lll}
x & x & x
\end{array}\right]^{T}
\end{gathered}
$$

According to ADS-B data update features, position, speed, height and other information is updated every second, the time period this paper selected to take is $1 \mathrm{~s}$.

\section{B. Filtering Processing}

Filtering can increase data's reliability, improve recognition rate of flight track, eventually improve the efficiency of track correction. This paper uses limiting filtering method, compare the current received value with previous correct data, these two value are subtracted and use the absolute vale of their difference

as comparison basis, if it's not greater than $\Delta v$, the received data is on the correct track, otherwise, the received data is on the wrong track. Details are as follows:

$$
n= \begin{cases}0(\text { correct }) & \left|v_{n}-v_{n-1}\right| \leq \Delta v \\ 1(\text { wrong }) & \left|v_{n}-v_{n-1}\right| \geq \Delta v\end{cases}
$$

In filtering, the meaning of $\Delta v$ in position information, speed, altitude and heading is different, so we use the value of $\mathrm{v}$ to determine whether the track value is correct in track processing.

\section{Reliability -related IndicatorsFigures and Tables}

ADS-B data reliability evaluation includes information update rate, position discrete degree, jump-point rate and misspoint rate. During the transmission of surveillance data, due to the noise interference of various factors and the analytical error existing in the process of data analysis, there may be some discrepancies between the real track data and the true track data, which is the position dispersion of track data. When the position dispersion exceeds a certain threshold, it can be considered that the corresponding track point is a jumping point.

\section{1) Calculation Principle of Data Leakage Rate}

As mentioned earlier, the ADS-B information update rate should be between 0.5 and 2 seconds in order to ensure a reduction in aircraft separation and operational safety under normal conditions, but in any case, in the correct condition, every 1 second should have the message information existed. Based on this principle, it can be determined that as long as there's message data existing every one second, whether it's position information or other information like flight number, the message has no leakage, If there's no message for one second, it is determined that the data is lost, and the message has one leak point, if there's no data for several seconds, it is determined that these pieces of data are lost, and the message has multiple leak point.

First, the number of ADS-B leak point is initialized to 0 , then extract the ADS-B message data of an aircraft in a period, and divide it into $\mathrm{m}$ range according to the distance from aircraft to ADS-B station, next, determine whether there's a leak point due to the position and altitude of the aircraft. If there's no track point received in $m$ consecutive system cycles, it's considered to have leak points, and add $m$ to the number of ADS-B leak point, If there are data points in consecutive cycles, continue to extract data until the time interval expires, finally, use the following equation to calculate.

$$
\mu\left(\text { Update }_{\text {rate }}\right)=\frac{\sum \text { ADSBdata }_{\text {missing }}}{\sum A D S B \text { data }}
$$

\section{2) Calculation Principle of Data Jump Point Rate}

The ADS-B jump point rate calculation is to analyze and process the anomaly of message data. It mainly deals with distorted discrete data, find out the distortion point from position report, and filter them. The determination of the mean square error in the Kalman filtering calculation is used as the judgment of the jump point rate of the message data. First extract a certain time period message information from ADS-B message, then use Kalman filtering algorithm to filter the ADS$B$ position message, if mean square error is larger than prescribed threshold value, it's determined that the track point is fake, AKA jump point, otherwise, it's determined that the track point is on the flight track so that it can be considered as correct surveillance information, meanwhile, count the number of correct point and fake point, calculate the percentage of fake point, draw the curve. 


\section{3) Calculation Principle of Data Dispersion}

As for the assessment of position dispersion, first, use filter algorithm to filter ADS-B track and get a smooth reference track, then compare it with real track, calculate the dispersion of track position information to get the dispersion of ADS-B track data.

First, extract a certain time period message information from ADS-B message, then use Kalman filtering algorithm to filter the ADS-B position message, calculate error between these two track points, doing statistic analysis about error mean and variance, draw the curve.

\section{SimUlation ANALYSiS}

\section{A. CV Model Filtering}

Set the target to do uniform linear motion, its initial position is $(0,0), T=1 \mathrm{~s}, \mathrm{v} 0=220 \mathrm{~m} / \mathrm{s}$, var= 10 , the simulation result is shown as FIGURE II. In FIGURE II, real trajectory is indicated in black line, the observation trajectory is indicated in blue line, and the filtering trajectory is indicated in red line. We can see that the filtering trajectory is close to the real trajectory, filtering effect is good.

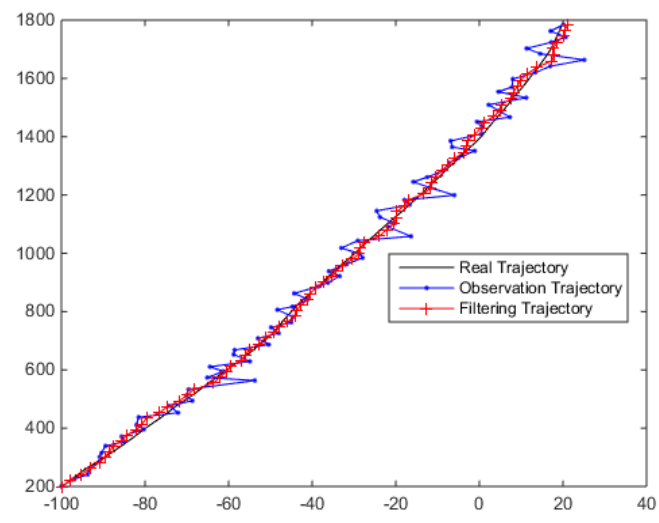

FIGURE II.

\section{MODEL FILTERING SIMULATION}

\section{B. CA Model Filtering}

Set the target to do uniform acceleration linear motion, its initial position is $(0,0), v x=180 \mathrm{~m} / \mathrm{s}, \mathrm{vy}=120 \mathrm{~m} / \mathrm{s}, \mathrm{T}=1 \mathrm{~s}$, the simulation result is shown as Figure 5.2. In Figure 5.2, real trajectory is indicated in black line, the observation trajectory is indicated in blue line, and the filtering trajectory is indicated in red line. we can see that the filtering trajectory is close to the real trajectory, filtering effect is good.

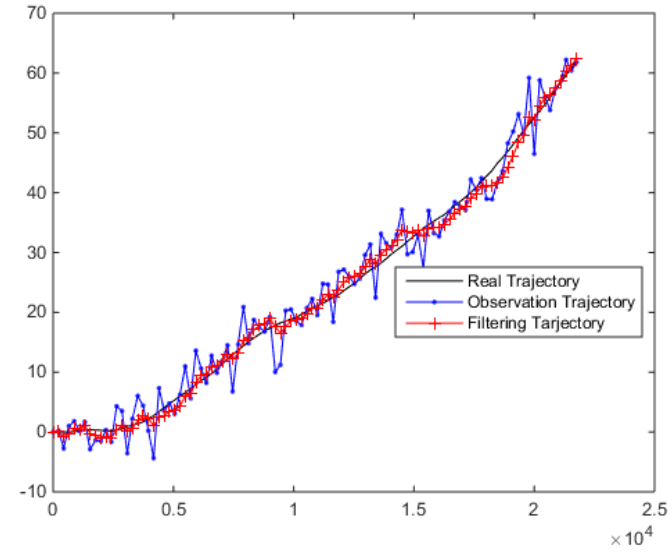

FIGURE III. CA MODEL FILTERING SIMULATION

\section{CONCLUSION}

This paper mainly introduces the concept and development status of ADS-B, discusses the advantages and disadvantages of ADS-B and the current main surveillance system - radar surveillance, and puts forward the idea of ADS-B information segmentation evaluation the reliability, integrity and accuracy of ADS-B information based on CV/CA Model. By using this evaluation method can we improve the efficiency of ADS-B surveillance, and predict the possible conflict, eventually improve flight safety.

\section{ACKNOWLEDGMENT}

The authors would like to thank the financial support by National Natural Science Foundation of China (No.61501225) and College Students' Free Exploration Program of Nanjing University of Aeronautics and Astronautics （ZT2016058）

\section{REFERENCES}

[1] China Civil Aviation ADS-B implementation plan" revised by Civil Aviation Authority [J]. China Civil Aviation, 2016(2):6-6.

[2] Tianyu Xu, Application and Development of ADS-B Technology in China Civil Aviation [J]. Jiangsu Aeronautics, 2012(4):62-64.

[3] Zilong Wang, Quality Analysis of ADS-B Surveillance [D]. China Civil Aviation Flight Academy, 2013.

[4] Wang, H. ADS-B used in improvement of air traffic control (Order No. 1598245). Available from ProQuest Dissertations \& Theses Global A\&I: The Sciences and Engineering Collection, 2015.

[5] Strohmeier M, Schafer M, Lenders V, et al. Realities and challenges of next gen air traffic management: the case of ADS-B[J]. IEEE Communications Magazine, 2014, 52(5):111-118.

[6] Shijia Tian, Xiaopeng Yang, Kun Yao. A New Data Fusion Algorithm for TCAS II and ADS - B [J]. Sensors and Microsystems, 2016, 35(3):130132.

[7] Guiping He. Research on Data Fusion Method of ADS - B and Radar Combination Surveillance [D]. China Civil Aviation Flight Academy, 2011.

[8] Shaoyue Zhang, Hailong Du, Chunyan Gao. Research on Correction of ADS-B Trajectory based on basic flight models. Journal of Civil Aviation University of China[J]. 2013(5):1-4.

[9] Man Hu. Flight Trajectory process and filtering based on ADS-B[D]. University of Electronic Science and Technology of China, 2012.

[10] Shuai Shao. Research on ADS-B Track Processing and Display Technology[D]. Harbin Engineering University, 2013. 